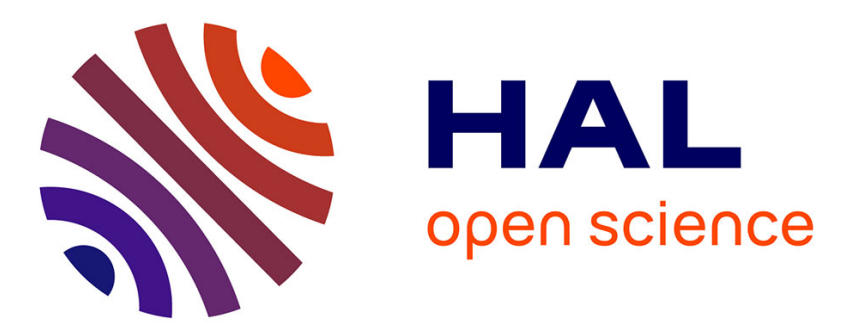

\title{
Influence of the geometry of illumination and viewing beams on displacement measurement errors in interferometric metrology
}

\author{
Ch. de Veuster, Pierre Slangen, Y. Renotte, L. Berwart, Y. Lion
}

\section{To cite this version:}

Ch. de Veuster, Pierre Slangen, Y. Renotte, L. Berwart, Y. Lion. Influence of the geometry of illumination and viewing beams on displacement measurement errors in interferometric metrology. Optics Communications, 1997, 143 (1-3), pp.95-101. 10.1016/S0030-4018(97)00336-2 . hal-02012248

\author{
HAL Id: hal-02012248 \\ https://hal.science/hal-02012248
}

Submitted on 8 Jun 2021

HAL is a multi-disciplinary open access archive for the deposit and dissemination of scientific research documents, whether they are published or not. The documents may come from teaching and research institutions in France or abroad, or from public or private research centers.
L'archive ouverte pluridisciplinaire $\mathbf{H A L}$, est destinée au dépôt et à la diffusion de documents scientifiques de niveau recherche, publiés ou non, émanant des établissements d'enseignement et de recherche français ou étrangers, des laboratoires publics ou privés. 


\title{
Influence of the geometry of illumination and viewing beams on displacement measurement errors in interferometric metrology
}

\author{
Ch. De Veuster ${ }^{\text {a }}$, P. Slangen ${ }^{\text {b }}$, Y. Renotte ${ }^{\text {a }}$, L. Berwart ${ }^{a}$, Y. Lion ${ }^{\text {a. } *}$ \\ "HOLOLAB. Laboratoire de Physique Générale, Institut de Physique B-5, B-4000 Liège I, Belgium \\ ${ }^{\mathrm{b}}$ ENSTIMA - LMM, Ecole Nationale Supérieure des Techniques Industrielles et des Mines d'Alès, Avi. de Clavières 6, F-30319 Alès, France
}

\begin{abstract}
Out-of-plane interferometers are widely used in metrological investigations. Most of them use divergent beams to allow displacement measurements on a large dimension object and have a configuration which makes them sensitive to in-plane displacements. The limits of such systems are described. The error for different interferometric configurations is computed and solutions to correct it are proposed. It must be noticed that the error could be very large for some common configurations and has to be corrected.
\end{abstract}

Kerwords: Speckle; Interferometry; Sensitivity; Error; Out-of-plane

\section{Introduction}

Interferometry is an easy and fast way to measure displacements or deformations of objects. Interferometric studies of mechanical structures enable one to compare displacement maps with calculated maps (e.g. with finite elements). Those methods are appreciated for non-destructive testing. Previous works have already studied errors related, among others, to phase-shifting algorithms, phaseshifter calibration or non-linearities [1-5]. Influence of systematic errors due to beam geometry has also been reported for speckle photography [6]. This study is concerned with out-of-plane sensitive interferometers. Some considerations about in-plane sensitive interferometers will also be presented.

In most DSPI (Digital Speckle Pattern Interferometry) and holographic interferometers the object is illuminated with a divergent beam which allows the study of large objects. However the use of a divergent illumination beam

\footnotetext{
${ }^{*}$ Corresponding author. E-mail: lion@gw.unipc.ulg.ac.be.
}

can lead to large errors for quantitative measurement of deformations. The geometry of both illumination and viewing beams influence displacement measurements whatever the type of interferometer (speckle, holographic, ... ) might be.

\section{Displacement measurements}

Usually the object is illuminated with a divergent beam by means of a spatial filter or an optical fibre (illumination point IP in Fig. 1). When the object is observed through an aperture, the object forms the base of a "viewing cone". The part of the viewing cone situated between the object and the entrance pupil of the observation system shows real beams. The actual paths of the beams in the recording system are not drawn but are supposed to converge to a point which we call OP (observation point in Fig. 1).

The general theory for determination of quantitative displacements can be found in the literature [7]. The sensitivity vector $\boldsymbol{K}$ of an interferometric system gives the measurement direction bisecting the illumination and the viewing beams. Its amplitude is $2 \pi / \lambda$. The phase variation $\Delta \Phi$ occurring during a displacement corresponds to 


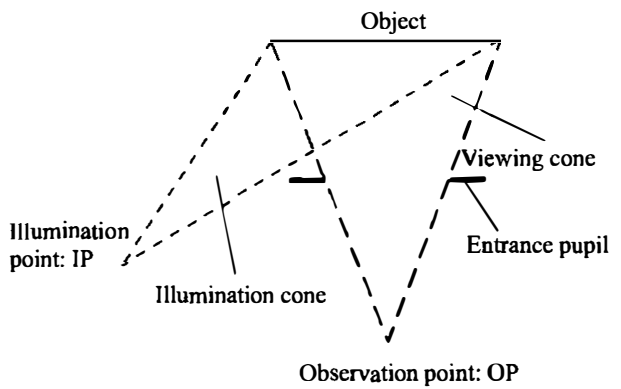

Fig. 1. General configuration for the illumination and observation of an object.

the projection of the displacement vector $\boldsymbol{D}$ on the sensitivity vector:

$\Delta \Phi=\boldsymbol{K} \cdot \boldsymbol{D}$.

The displacement modulus $D$ is extracted from Eq. (1). For example if the illumination and viewing beams make equal angles with the surface normal [7]:

$D=\frac{\Delta \Phi \lambda}{4 \pi \cos \alpha}$,

where $\lambda$ is the wavelength and $\alpha$ is half the angle between the illumination and the viewing beams.

The drawback of such measurements is that the sensitivity vector direction changes as illumination and viewing beam directions change. Then the sensitivity varies from point to point and the displacement measurement requires the accurate knowledge of illumination, observation and object point positions. A solution to that problem consists of using an out-of-plane sensitive interferometer. Such an interferometer could be realized by illuminating and viewing the object in a direction normal to the object surface or by oblique illumination and observation (the illumination and observation directions making equal and opposite angles with the surface normal) [7].

Most of actual systems have another configuration due to the current need to investigate large dimension objects and to make compact interferometric set-ups. In that configuration the object is illuminated with a divergent beam making a non-zero angle with the sensitivity direction. Such a system was described for an object with the normal to its surface aligned with the optical axis of the observation system [8]. In that case the measured displacement is given by:

$D=\frac{\Delta \Phi \lambda}{2 \pi(1+\cos \beta)}$,

where $\Delta \Phi$ is the phase variation occurring during the displacement, $\lambda$ is the wavelength and $\beta$ is the angle between the illumination direction and the normal to the object surface. The sensitivity (coefficient of the phase variation $\Delta \Phi$ ) decreases as $\beta$ increases (Eq. (3)).

An approximation is made when such configurations are used. We will present the limits of the approximation for quantitative metrology.

\section{Sensitivity error}

Let us generalize the sensitivity error investigations to oblique illumination and viewing directions. The out-ofplane interferometer sensitivity decreases when the illumination angle $\theta_{i}$ (viewing angle $\theta_{i}^{\prime}$ ) increases. Fig. 2 shows that the sensitivity at a point $P$ changes during displacement $\left(\theta_{2}\right.$ versus $\theta_{1}$ and $\theta_{2}^{\prime}$ versus $\left.\theta_{1}^{\prime}\right)$ and also that the sensitivity changes from point to point when the object is at rest $\left(\theta_{i}\right.$ as a function of $\left.B\right)$.

The optical path length measured at point $\mathrm{P}$ before displacement (position I in Fig. 2) is given by:

$$
\frac{A}{\sin \theta_{1}}+\frac{A^{\prime}}{\sin \theta_{1}^{\prime}},
$$

and the optical path length measured at $\mathrm{P}$ after a displacement $D$ (position II in Fig. 2) is given by:

$$
\frac{A+D}{\sin \theta_{2}}+\frac{A^{\prime}+D}{\sin \theta_{2}^{\prime}} \text {. }
$$

The Optical Path Difference (OPD) measured at $\mathrm{P}$ for a displacement $D$ in the $z$ direction (Fig. 2) is given by subtracting Eq. (4) from Eq. (5):

$\mathrm{OPD}_{\mathrm{P}}=\left(\frac{A+D}{\sin \theta_{2}}+\frac{A^{\prime}+D}{\sin \theta_{2}^{\prime}}\right)-\left(\frac{A}{\sin \theta_{1}}+\frac{A^{\prime}}{\sin \theta_{1}^{\prime}}\right)$.

The OPD at $\mathrm{P}$ for a displacement $D$ when constant sensitivity is assumed (illumination and viewing angles do not change during displacement) is given by [7]:

$\mathrm{OPD}_{\mathrm{P}}^{\text {const }}=D\left(\sin \theta_{1}+\sin \theta_{1}^{\prime}\right)$.

Angles $\theta_{1}, \theta_{2}, \theta_{1}^{\prime}$ and $\theta_{2}^{\prime}$ are given by:

$\tan \theta_{1}=\frac{A}{B}, \quad \tan \theta_{2}=\frac{A+D}{B}, \quad \tan \theta_{1}^{\prime}=\frac{A^{\prime}}{B^{\prime}}$,
$\tan \theta_{2}^{\prime}=\frac{A^{\prime}+D}{B^{\prime}}$.

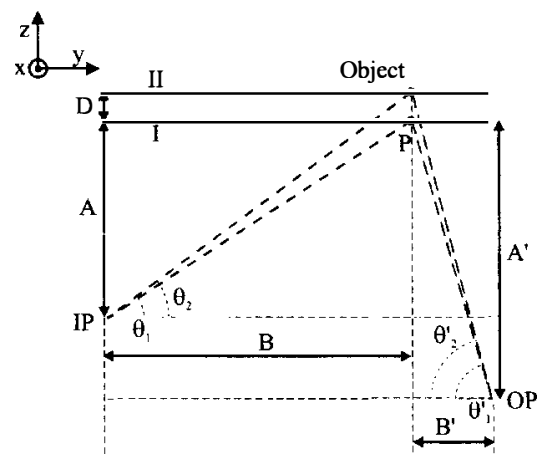

Fig. 2. Change in sensitivity due to an object displacement from position I to position II. 


\section{Sensitivity emor $\left(A=A^{\prime}=B=B^{\prime}=0,1 \mathrm{~m}=>\theta_{i}=\theta_{i}{ }^{\prime}=45^{\circ}\right)$}

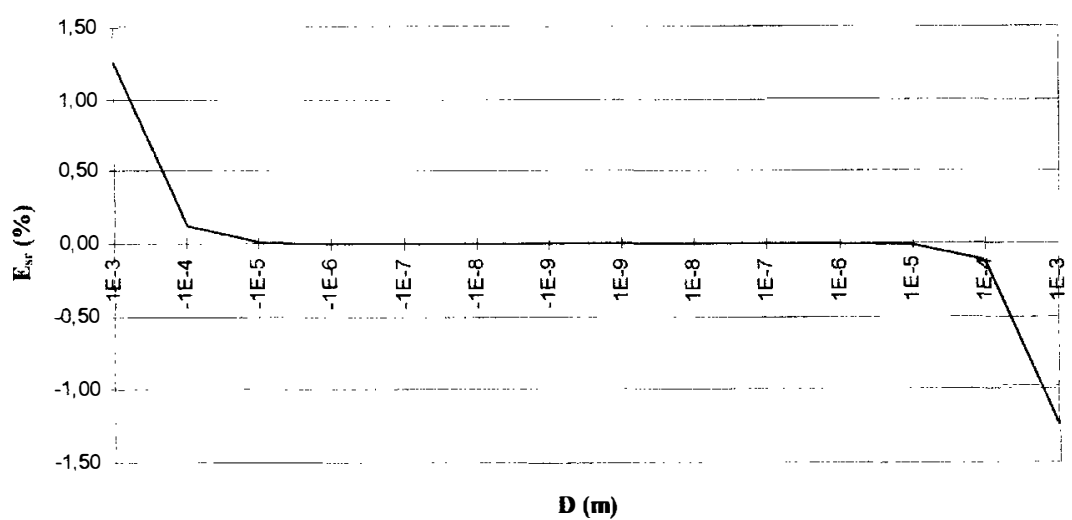

Fig. 3. Influence of the out-of-plane displacement $D$ on the sensitivity error $E_{\mathrm{sr}}$ for illumination and observation angles of $45^{\circ}$.

The sensitivity error $E_{\varsigma}$ at $\mathrm{P}$ is:

$$
E_{\mathrm{s}}=\mathrm{OPD}_{\mathrm{P}}^{\text {const }}-\mathrm{OPD}_{\mathbf{p}} \text {. }
$$

Substituting Eqs. (6), (7) and (8) in (9), the sensitivity error becomes:

$$
\begin{aligned}
E_{\mathrm{s}}= & D\left(\frac{1}{\sqrt{1+(B / A)^{2}}}+\frac{1}{\sqrt{1+\left(B^{\prime} / A^{\prime}\right)^{2}}}\right. \\
& \left.-\sqrt{1+\left(\frac{B}{A+D}\right)^{2}}-\sqrt{1+\left(\frac{B^{\prime}}{A^{\prime}+D}\right)^{2}}\right) \\
& -A\left(\sqrt{1+\left(\frac{B}{A+D}\right)^{2}}-\sqrt{1+\left(\frac{B}{A}\right)^{2}}\right) \\
& -A^{\prime}\left(\sqrt{1+\left(\frac{B^{\prime}}{A^{\prime}+D}\right)^{2}}-\sqrt{1+\left(\frac{B^{\prime}}{A^{\prime}}\right)^{2}}\right) .
\end{aligned}
$$

Eq. (10) divided by Eq. (7) leads to the relative sensitivity error at $P$ :

$$
E_{\mathrm{sr}}=\frac{E_{\mathrm{s}} \sqrt{1+(B / A)^{2}} \sqrt{1+\left(B^{\prime} / A^{\prime}\right)^{2}}}{D\left(\sqrt{1+(B / A)^{2}}+\sqrt{1+\left(B^{\prime} / A^{\prime}\right)^{2}}\right)} .
$$

The same equations hold for an object displacement $D$ in the opposite direction of axis $z$. In that case $D$ is negative. Notice that illumination and observation variables are related together. As a matter of fact $B+B^{\prime}$ and $A^{\prime}-A$ are constant. Angles $\theta_{1}, \theta_{2}, \theta_{1}^{\prime}$ and $\theta_{2}^{\prime}$ are related together via $A, A^{\prime}, B$ and $B^{\prime}$ as shown in Eq. (8).

The relative sensitivity error $E_{\mathrm{sr}}$ depends on the object displacement $D$. That dependence versus $D$ is due to the illumination (viewing) angle variation during displacement and to the difference of sensitivity between different points. Hopefully Fig. 3 shows that the displacement $D$ does not affect significantly the sensitivity error $E_{\mathrm{sr}}$ when its value remains less than one millimetre $\left(E_{\mathrm{sr}}<1.5 \%\right)$. Error $E_{\mathrm{sr}}$

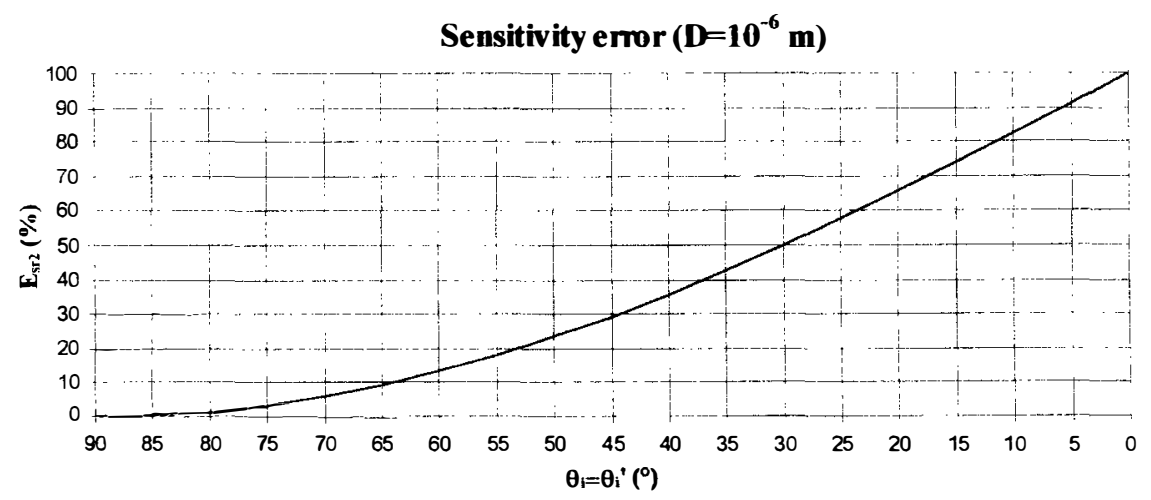

Fig. 4. Sensitivity error $E_{\mathrm{sr}}$ as a function of illumination $\theta_{i}$ and observation $\theta_{i}^{\prime}$ angles. The values are computed for $\theta_{i}=\theta_{i}^{\prime}$. 
due to sensitivity variation during displacement increases with both illumination and viewing angles. The curve of Fig. 3 is computed for $\theta_{i}$ and $\theta_{i}^{\prime}$ equal to $45^{\circ}$ which could be considered as an extreme experimental case. Such large illumination angles are used when the object is near IP or OP for the study of objects with large dimensions.

Eq. (11) provides the sensitivity error due to a displacement $D$ at a point $\mathrm{P}$. Another sensitivity error is due to the difference of sensitivity between object points. That error is estimated by comparison of the OPD due to a displacement $D$ (Eq. (6)) at different points:

$E_{\mathrm{sr} 2}=\frac{\mathrm{OPD}_{\mathrm{P} 1}-\mathrm{OPD}_{\mathrm{P} 2}}{O \mathrm{OD}_{\mathrm{P} 1}}$

In Eq. (12), P1 is considered as a reference point. We choose that point so that $\theta_{i}=\theta_{i}^{\prime}=0$, but it should be noticed that the choice of another reference point can increase or decrease the sensitivity error $E_{\mathrm{sr} 2}$. Fig. 4 shows that error $E_{\mathrm{sr} 2}$ increases with the illumination $\theta_{i}$ (viewing $\left.\theta_{i}^{\prime}\right)$ angle. That error must be corrected because it remains large even for small illumination or viewing angles. It should be noticed that the measured displacements are underestimated (Eq. (12) and Fig. 4) so that their use without correction can lead to dramatic miscalculations in mechanical structure design.

The error $E_{\mathrm{sr} 2}$ could be corrected by collimating both illumination and viewing beams or by numerical processing. The viewing beam is considered collimated when the object is far away from the observation system or when the viewing field has the same size as the entrance pupil of the observation system so that all viewing beams could be considered parallel.

Numerical processing consists of multiplying the measured displacement at point $\mathrm{P}$ by a sensitivity coefficient
$\mathrm{SC}^{\mathrm{P}}$ which is a function of illumination and viewing angles (IP, OP and $P$ positions):

$\mathrm{SC}^{\mathrm{P}}=\frac{100+E_{\mathrm{sr} 2}^{\mathrm{P}}}{100}$

The sensitivity error $E_{\mathrm{sr} 2}$ could be numerically corrected if the displacement $D$ is only out-of-plane as shown in Section 5. This error must absolutely be compensated for applications requiring qualitative (fringes counting) or quantitative measurements when illumination or viewing angles exceed $5^{\circ}$ (Fig. 4).

Among both solutions to correct sensitivity errors due to illumination and viewing cones, the use of collimated beams is the best because one does not need to know for every tested object the accurate position of any of the following points: illumination point, observation point and each object point. Such processing becomes very tedious when series of measurements must be achieved for a lot of objects placed at different positions.

\section{Influence of object shape}

All errors mentioned in the previous section are related with the object shape (via $A^{\prime}$ and $A$ ). So the sensitivity error $E_{\mathrm{sr} 2}$ at a given pixel will vary from one object to another and it will also vary from one pixel to another for a given object shape. A height difference of one centimetre can strongly modify the results of displacement measurements (Fig. 5 and Eq. (10)). Such height differences are frequently encountered for common mechanical pieces (e.g. turbine blades)

From Fig. 5 it can be shown that the error increases with the illumination angle (versus $B$ for $A$ constant). The

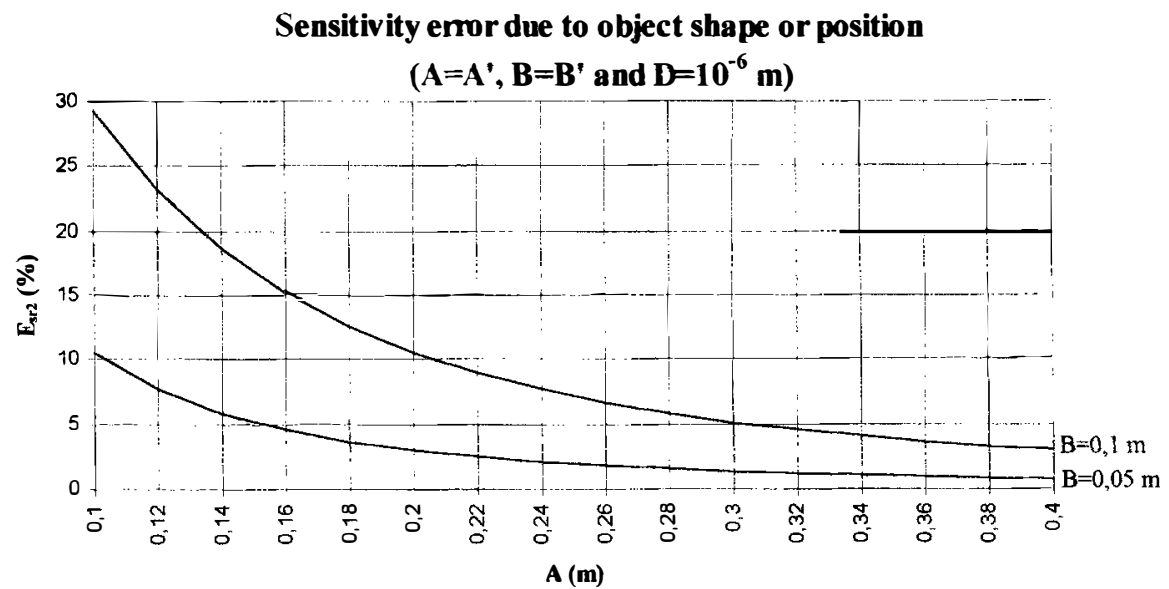

Fig. 5. The shape and position of the object can strongly modify displacement measurements. Height differences (range between two values of the horizontal axis) lead to large error differences (difference between the corresponding values on the vertical axis). 

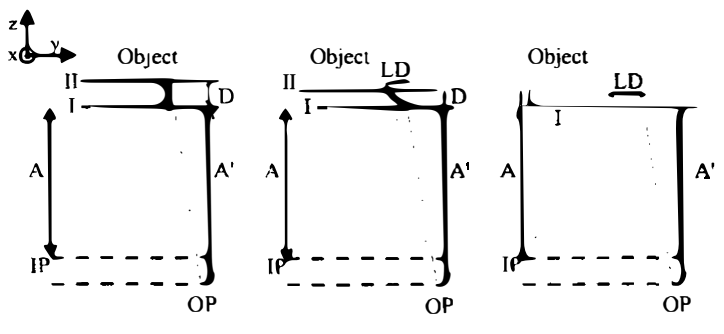

a

b

c

Fig. 6. (a) Displacement only out-of-plane. (b) Combined out-ofplane and in-plane displacement. (c) Displacement only in-plane.

sensitivity error $E_{\mathrm{sr} 2}$ due to the object shape can also be corrected by numerical processing if the object shape is known a priori. In that case pre-processing becomes very expensive in computing time because the object shape and the distance between the object and the observation system (illumination point) must be measured before any displacement measurement. The illumination and viewing beams collimation is a faster and more reliable solution to correct the error although it does not allow displacement measurements of a large object in one step. Nevertheless displacements can be measured by scanning the object with the collimated beam.

\section{In-plane sensitivity of a frequently used out-of-plane sensitive interferometer}

The use of a divergent illumination (viewing) beam leads to in-plane sensitivity in out-of-plane sensitive interferometers.

In previous sections the object was assumed to move only in the $z$ direction (out-of-plane displacements). In most structure behaviour tests a stressed object surface moves simultaneously out-of-plane and in-plane. The three displacements shown in Fig. 6 produce the same phase variation. That phase variation can be produced by an out-of-plane displacement (Fig. 6a), a combination of outof-plane and in-plane displacements (Fig. 6b) and a displacement which is completely in-plane (Fig. 6c). During a three-dimensional displacement it becomes impossible to extract displacement components and therefore to know the direction of the displacement.

Introducing the lateral displacement $\mathrm{LD}$ as the projection on the object plane of the total displacement, the in-plane sensitivity error $E_{\mathrm{sr}}$ can be calculated by Eq. (10). Angles $\theta_{2}$ and $\theta_{2}^{\prime}$ become:

$\tan \theta_{2}=\frac{A}{B \pm \mathrm{LD}}, \quad \tan \theta_{2}^{\prime}=\frac{A^{\prime}}{B^{\prime} \pm \mathrm{LD}}$.

Substituting Eq. (14) in Eq. (10) yields the sensitivity error:

$$
\begin{aligned}
E_{\mathrm{sLD}}= & D\left(\frac{1}{\sqrt{1+(B / A)^{2}}}+\frac{1}{\sqrt{1+\left(B^{\prime} / A^{\prime}\right)^{2}}}\right. \\
& \left.-\sqrt{1+\left(\frac{B \pm \mathrm{LD}}{A+D}\right)^{2}}-\sqrt{1+\left(\frac{B^{\prime} \pm \mathrm{LD}}{A^{\prime}+D}\right)^{2}}\right) \\
& -A\left(\sqrt{1+\left(\frac{B \pm \mathrm{LD}}{A+D}\right)^{2}}-\sqrt{1+\left(\frac{B}{A}\right)^{2}}\right) \\
& -A^{\prime}\left(\sqrt{1+\left(\frac{B^{\prime} \pm \mathrm{LD}}{A^{\prime}+D}\right)^{2}}-\sqrt{1+\left(\frac{B^{\prime}}{A^{\prime}}\right)^{2}}\right) .
\end{aligned}
$$

Eq. (15) divided by Eq. (7) gives the relative error:

$$
E_{\mathrm{sLDr}}=\frac{E_{\mathrm{sLD}} \sqrt{1+(B / A)^{2}} \sqrt{1+\left(B^{\prime} / A^{\prime}\right)^{2}}}{D\left(\sqrt{1+(B / A)^{2}}+\sqrt{1+\left(B^{\prime} / A^{\prime}\right)^{2}}\right)} \text {. }
$$

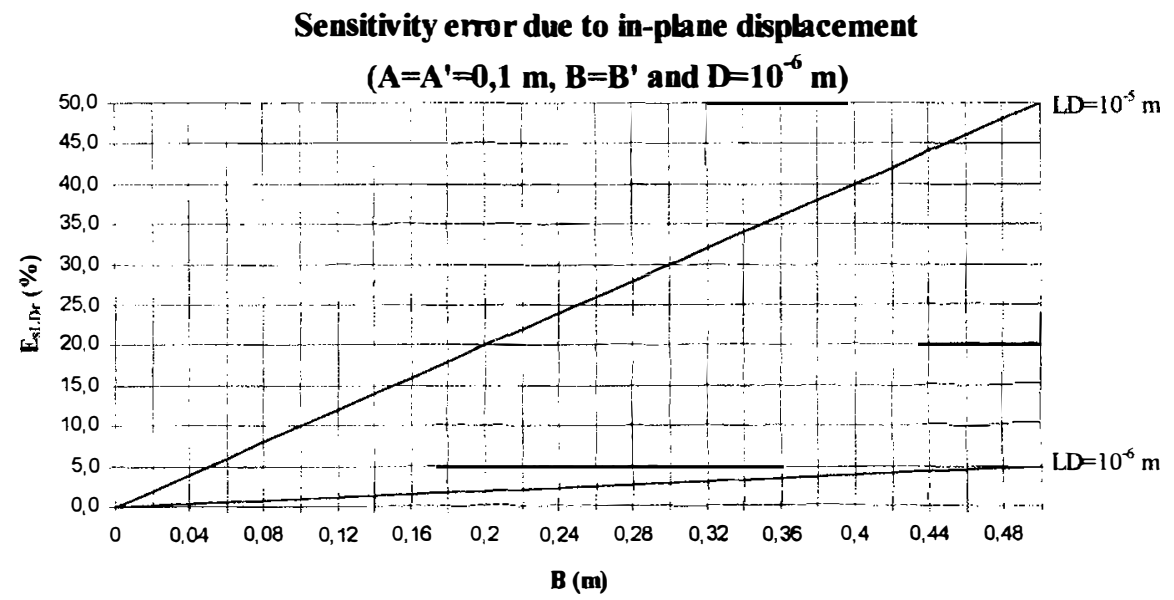

Fig. 7. Influence of in-plane displacement to out-of-plane measurements. The sensitivity error $E_{\mathrm{sLDr}}$ versus the illumination (viewing) angle. The lateral component of the displacement (LD) is subtracted from $B$ and $B^{\prime}$ (Eq. (15)). 
Sensitivity error due to in-plane sensitivity

$\left(A=A^{\prime}=0,1 \mathrm{~m}\right.$ and $\left.B=B^{\prime}=0,1 \mathrm{~m}\right)$

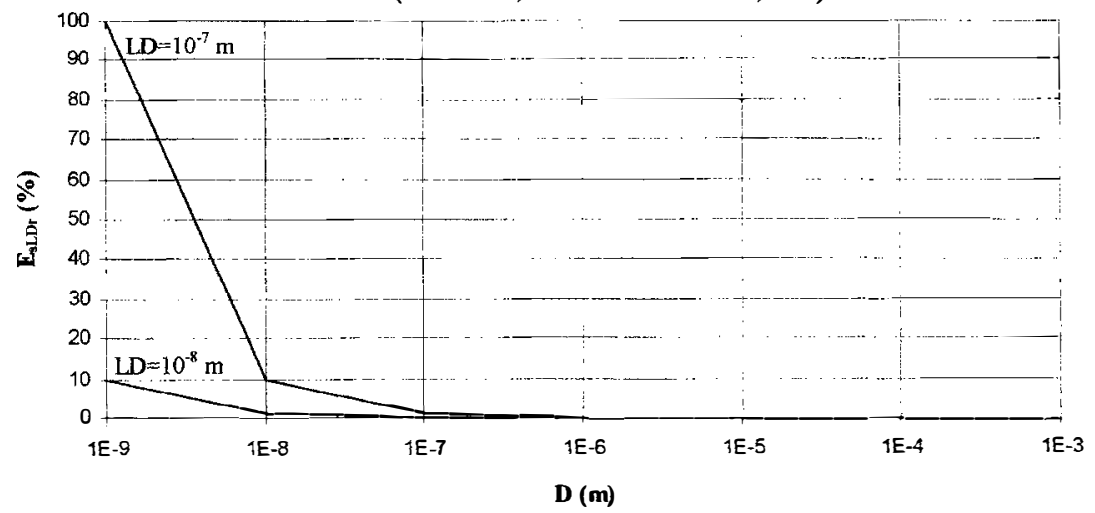

Fig. 8. Influence of in-plane displacement to out-of-plane measurements. The sensitivity error $E_{\mathrm{sLDr}}$ versus the out-of-plane displacement $D$. The lateral component of the displacement (LD) is substracted from $B$ and $B^{\prime}$ (Eq. (15)).

Fig. 7 shows that the error $E_{\mathrm{sLDr}}$ can be very large even for small lateral displacements (microdisplacements) and also for small illumination and viewing angles $\left(<5^{\circ}\right.$ i.e. for $B$ or $B^{\prime}<0.01 \mathrm{~m}$ ). Eq. (15) could be written in four ways adopting a different sign for the two directions of the lateral displacement LD. Each equation relates to different relative positions of IP, OP and the object point P. Fig. 7 shows data for an out-of-plane displacement $D$ in the $z$ direction and a lateral displacement in the direction opposite to $y$. In that case the displacement is underestimated. A lateral displacement in the direction of $y$ would lead to an overestimated out-of-plane displacement. In both figures IP and OP are positioned at the same side versus $P$. The error increases linearly as a function of $B\left(=B^{\prime}\right)$, i.e., for increasing illumination (viewing) angles.

Fig. 8 shows that the error $E_{\mathrm{sLDr}}$ decreases when the displacement $D$ increases. The larger the displacement $D$ the less sensitive the error $E_{\mathrm{sLDr}}$ will be to lateral displacement LD. The error for any lateral displacement decreases with exponential rate. It can be noticed that the out-of-plane displacement $D$ must be hundred times greater than the lateral displacement to neglect the error. If the in-plane displacement is ten times greater than the out-of-plane displacements, the error due to LD (Fig. 8) is about $10 \%$.

In DSPI, the in-plane sensitivity is limited by speckle decorrelation. The largest error is produced when the in-plane displacement value is equal to the speckle size (usually about fifteen micrometers) and no information is obtained when a larger in-plane displacement occurs. Larger speckles allow a greater lateral displacement before decorrelation. In interferometric holography, the coherence length of the laser is the only limit to that in-plane sensitivity.

The large error $E_{\mathrm{sLDr}}$ induced by the lateral displacement must also be compensated. In this case there is no numerical processing able to correct $E_{\mathrm{sLDr}}$ because it is impossible to distinguish out-of-plane displacements, inplane displacements and the sensitivity errors $E_{\mathrm{sr} 1}$ and $E_{\mathrm{sr} 2}$ from phase variation measurements. The only solution is to collimate both illumination and viewing beams. But that is still not enough because the interferometer remains in-plane sensitive if the illumination and viewing angles are nonzero (Fig. 9). The phase variation $\Delta \Phi$ is due to lateral displacement. The in-plane component of the sensitivity direction is given by the intersection of the object plane and the plane including both illumination and viewing beams. The complete and more useful solution is to align illumination and viewing collimated beams $\left(B=B^{\prime}=0\right)$. So if illumination and viewing angles are equal to zero the interferometer is only out-of-plane sensitive. Angles ranging between zero and ninety degrees achieve a balance between out-of-plane and in-plane sensitivity.

The in-plane sensitivity does not allow correct out-ofplane measurements. Hopefully in most cases the in-plane displacement is negligible regardless of the out-of-plane displacement. For example for the tilt of a one centimetre long plate the out-of-plane maximum displacement has to be two hundred micrometers before the in-plane component disturbs the measurements. In our experimental set-up [9] such a displacement is monitored in ten successive data

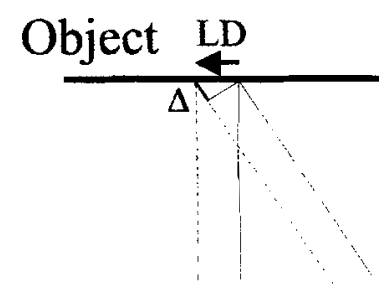

Fig. 9. Phase variation $\Delta \Phi$ induced by an in-plane displacement of the object when collimated beams are used. 
acquisitions each containing about thirty fringes. This limits the measurement of large displacements.

The in-plane sensitivity does not allow displacement measurements of vibrating objects in free-free mode (hanging object) or with consistent in-plane vibrations.

\section{3-D displacement measurements and in-plane sensi- tive interferometer considerations}

Measurement of 3-D displacements could be realized using three collimated out-of-plane sensitive interferometers which have non-coplanar sensitivity directions [7]. The collimation of the beams ensures the recording of reliable measurements. Each interferometer records the projection of out-of-plane and in-plane displacements following a sensitivity direction.

Another solution consists of using one out-of-plane sensitive interferometer and two in-plane sensitive interferometers. The in-plane sensitive interferometers illuminate the object with two beams located in the same plane and having the same angle with the optical axis of the observation system. Most commercial and laboratory set-ups use divergent beams to illuminate the object. Some systematic errors similar to those reported above for the out-of-plane interferometer could be corrected by numerical processing but to cancel all errors the two illumination beams and "the viewing beam" must be collimated. A very important error is related to divergent illumination which produces out-of-plane sensitivity of the in-plane interferometer. As a matter of fact when divergent beams illuminate the object, the angles between each illumination beam and the optical axis are not equal. That error cannot be corrected by numerical processing because it is not possible to separate in-plane and out-of-plane displacements. So the only way to record reliable in-plane displacement measurements is to collimate all the beams.

\section{Conclusion}

We have pointed out displacement measurement errors due to the geometry of illumination and viewing beams. The in-plane sensitivity error of a so-called out-of-plane sensitive interferometer was investigated and corrected. All those errors are due to sensitivity variations at different object points or to sensitivity variations induced by displacements. Those errors which appear in most interferometric set-ups must be corrected to ensure the reliability of qualitative or quantitative measurements.

Sensitivity variations between object points must be compensated using collimated beams. In that case, the interferometric set-up would allow accurate quantitative measurements for every object position, every object shape, any displacement even large displacements and any kind of application (profilometry, vibration or surface measurements, static or quasi-static displacements, 3-D measurements, ...). All above considerations are also true for convergent beams. All investigations were carried out and verified for phase-shifted digital speckle pattern interferometry. Distance or displacement measurements over large objects could be achieved by scanning the object with collimated beams.

\section{Acknowledgements}

Ch. De Veuster wishes to thank the FRIA (Fonds pour la formation à la Recherche dans l'Industrie et l'Agriculture) for financial support. The authors thank the reviewers for their constructive remarks and helpful suggestions.

\section{References}

[1] J. Schwider, R. Burow, K.-E. Elssner, J. Grzanna, R. Spolaczyk, K. Merkel. Appl. Optics 22 (1983) 3421.

[2] K. Creath, P. Hariharan, Appl. Optics 33 (1994) 24.

[3] K. Creath, P. Hariharan. Appl. Optics 33 (1994) 2585

[4] C. Joenathan. Appl. Optics 33 (1994) 4147.

[5] K. Hibino, B.F. Oreb, D.I. Farrant, K.G. Larkin, J. Opt. Soc. Am. A 12 (1995) 761.

[6] M. Sjödahl, L.R. Benckert, Appl. Optics 33 (1994) 7461.

[7] P.K. Rastogi, Ed., Holographic Interferometry: principles and methods, Springer Series in Optical Sciences (Springer, Berlin. 1994).

[8] R. Jones, C. Wykes, Eds., Holographic and Speckle Interferometry, Cambridge Studies in Modem Optics (Cambridge University Press, Cambridge, 1989).

[9] P. Slangen, C. De Veuster, Y. Renotte, L. Berwart, Y. Lion, Opt. Eng. 34 (1995) 3526. 